\title{
CAPÍTULO 6bis \\ Los nuevos tratados comerciales y las administraciones públicas
}

Julio V. GONZÁLEZ GARCÍA*

Abstract: There is currently an implosion of Free Trade Agreements: TTIP, CETA, RCEP. But their evolution extends its scope in administrative fields. The right to legislate, the impact of ISDS in comparison with the judicial control of the Public administration or even more, the impact in the Social and Democratic State are new questions of the Free Trade that are analyzed in the present chapter.

\section{PLANTEAMIENTO GENERAL}

Las negociaciones que están manteniendo desde febrero de 2014 la Unión Europea y los Estados Unidos para la adopción del TTIP (Transatlantic Trade and Investment Partnership) y la controversia política que ha provocado han servido para llevar a la opinión pública uno de los cambios más importantes en el funcionamiento de las Administraciones Públicas: el impacto de los Tratados de Nueva Generación.

* Catedrático de Derecho administrativo. Director del Instituto de Derecho Europeo e Integración Regional Universidad Complutense de Madrid. E-mail: julgonza@ ucm.es https://www.globalpoliticsandlaw.com. Este artículo se enmarca en el Proyecto de Investigación DER2016-76986-P; «Unión Europea en el contexto de los Tratados de nueva generación: entre reforma institucional y protección social», del que soy IP. 
No es algo que derive o no de que, finalmente el TTIP llegue a buen puerto o no. Los Estados han ido desarrollando, más allá de factores políticos coyunturales, un nuevo modelo de tratados comerciales que traspasa su formulación tradicional de regular aranceles y otros elementos esencialmente comerciales y que se adentran en aspectos que son propios de la regulación administrativa y que, incluso, tienen una impronta constitucional ya que afecta a aspectos esenciales del régimen jurídico del control judicial de la Administración (artículo $106 \mathrm{CE}$ ) o de las garantías democráticas (artículo 1.1 y artículos concordantes). En mi opinión, están aquí para quedarse.

La razón posiblemente estribe en que constituyen un elemento en el desarrollo del capitalismo que, triunfante tras la desaparición de la Unión Soviética en 1991, ansía establecer unas reglas económicas comunes para todo el mundo. La globalización económica tuvo un impacto general en el funcionamiento de los entes públicos introduciendo ideas desconocidas como las de la competencia entre entes públicos, que se favorecieron con la desregulación y la liberalización. Aunque siempre existió la tentación de aprobar acuerdos más generales, que involucraran a la mayor parte de los países para crear un espacio económico y político global.

Hace unos años, se señaló que el Acuerdo Multilateral de Inversiones constituían la "Constituci de una economía global única», por recoger la expresión del antiguo Secretario General de la Organización Mundial de Comercio, Renato Ruggero. El fracaso de aquel tratado hizo modificar los instrumentos a través de los cuales se impulsaba la articulación de reglas económicas comunes. Y, en este sentido, conforman la manifestación más acabada del «Derecho administrativo global» ${ }^{1}$ del que se lleva hablando desde hace tiempo.

Por ello, de lo que se está hablando en la actualidad es de un fenómeno que va más allá del TTIP e incluso de Europa. De hecho, no debemos establecer una relación directa entre Trump y la paralización de las negocia-

1 Sobre el Derecho administrativo global, es recomendable la lectura de B. Kingsbury, B. y R.B. Stewart, R.B: Hacia el Derecho administrativo global: Fundamentos, Principios y Ambito de Aplicación, Global Law Press, Sevilla (2016). 
ciones sobre el TTIP debido a que la propia Casa Blanca ha señalado que aún no tiene posición. De hecho, sigue habiendo conversaciones (aunque no sean rondas negociadoras), que han permitido acercar posiciones en el complejo mercado farmacéutico.

La Unión Europea ha suscrito el CETA con Canadá y otros tratados de filosofía parecida con Vietnam, Singapur o Corea del Sur. Hemos abierto relaciones con México y Perú. Es previsible que tengamos que suscribir algo similar con el Reino Unido cuando se materialice el Brexit. Y Europa está ahora en medio del proceso de negociación del Trade in Services Agreement, que posiblemente sea el que más impacto va a tener en nuestras vidas como ciudadanos por afectar a los servicios públicos.

Pero en América, los restos del TPP abortado por Trump quieren reabrir la cuestión posiblemente para acercarlo al gran acuerdo comercial que están impulsando China, la India y Japón, el Regional Comprehensive Economic Partnership. Las negociaciones que se van a abrir sobre el rediseño del NAFTA nos hacen ver que sigue siendo un modelo que es tenido en cuenta.

\section{2. ¿POR QUÉ SON ESPECIALES LOS NUEVOS TRATADOS COMERCIALES?}

Estos nuevos Tratados marcan un antes y un después en la regulación comercial internacional. La razón esencial que motiva calificarlos como especiales, pudiendo ser considerados como Tratados de Nueva Generación, deriva del hecho de que superan el contenido usual de tipo de acuerdos internacionales y han pasado a incorporar reglas de regulación económica. Al mismo tiempo, constituyen reglas que estructuran un punto de partida para dicha integración económica, afectando de forma general al funcionamiento de las Administraciones Públicas.

En efecto, desde un primer punto de vista, hay que señalar de forma clara que se integran en el funcionamiento público y regulan elementos tanto de lo que puede ser la "parte general» del funcionamiento público (como ocurre, a título de ejemplo, con el capítulo 19 del CETA, referido a contratación 
pública ${ }^{2}$ que introduce reglas especiales que complementan las Directivas europeas e incluso la futura Ley de Contratos del Sector Público). Al mismo tiempo, contienen los elementos básicos de la regulación sustantiva de otros sectores, esto es, abordan lo que serían sectores concretos de Derecho administrativo económico. Y, obviamente, hay elementos puramente comerciales.

Con lo anterior, se destaca un aspecto importante, pero en mi opinión, no el más destacado. El régimen que se acaba de señalar constituirá el régimen mínimo del tratamiento del nacional, con independencia de la salvedad que diré con posterioridad en relación con la protección de inversiones. Dicho de otro modo, no sirve sólo para articular un régimen «entre socios internacionales», sino que van a permitir el desplazamiento de la normativa nacional. Sobre ello volveré con posterioridad.

El segundo factor por el que me parece que son muy relevantes y, a la par, diferentes, deriva del hecho de que tienen un contenido constitucional. En efecto, desde una perspectiva estatal, lo más relevante, de estos Tratados de nueva generación es su fuerza constitucional que incluye la capacidad de alterar reglas sin necesidad de modificar las Constituciones de los países en los que se va a aplicar. Una fuerza constitucional en todo lo que afecta a la ordenación de las actividades económicas y a cómo se articulan en la práctica los instrumentos de regulación y de resolución de controversias.

Aunque volveré sobre ello, planteo ya un interrogante, ¿cómo articulamos los mecanismos de protección de inversiones con la exigencia de control judicial de la Administración que está recogido en el artículo 106 de nuestro texto constitucional? ¿Nos encontramos ante una nueva manifestación de mutación constitucional o directamente se trata de una inconstitucionalidad que dejaría sin aplicación los arbitrajes de inversiones en nuestro país?

Esto no es un problema de nacionalismo ni de proteccionismo. La valoración política se puede hacer en otro momento y se podrá ver que los

2 Una exposición general del impacto de CETA en la contratación pública se puede ver en mi estudio «Contratación Pública en el Tratado de Libre Comercio entre la Unión Europea y Canadá», que se puede obtener en https://www.globalpoliticsandlaw.com/2017/03/12/ceta-y-contratos-publicos/ 
problemas que se plantea derivan de los valores internos que defiende, no si es más o menos proteccionista. Aquí de lo que se trata es de conciliar las reglas de estos tratados con lo que dispone nuestra Constitución. Porque pudiera ocurrir que la contravinieran.

$\mathrm{Y}$, pese a la importancia que tienen los elementos anteriores, hay que resaltar otro aspecto que, pare mí les dota de un peso suplementario: estos Tratados comerciales de nueva generación no son un punto de llegada sino de partida.

Disponen de elementos esenciales para la regulación económica que les permitirán modificar y extender su regulación: son los mecanismos de cooperación regulatoria que, desde una perspectiva democrática (que está en el artículo 1.1 del texto constitucional) nos va a hacer añorar los modos de regular de la Unión Europea a pesar de todos los problemas que plantean. Sobre ello volveré también más adelante. $Y$ esto nos va a cuestionar sustancialmente el contenido de nuestra regulación y la capacidad de articular democráticamente las oscilaciones legislativas, en el marco de un Estado social y democrático.

\section{FUNDAMENTO Y CONSECUENCIAS: UNA PERSPECTIVA EUROPEA}

La perspectiva que han adoptado estos tratados es comercial. Es el fundamento del que se ha partido a la hora de su articulación y que ha atraído el resto de los contenidos, estén o no vinculados. En el caso europeo es especialmente claro, ya que el impulso a los Tratados de Nueva Generación se produce como consecuencia de la promulgación del Tratado de Lisboa, donde se reconoce por vez primera de forma expresa la política comercial como una de las políticas europeas en el aparentemente modesto Título II de la Quinta Parte del Tratado, artículos 206 y 207.

El reconocimiento de su base comercial es muy relevante tanto desde un punto de vista formal como desde el del contenido que tienen estos Tratados de Nueva Generación y cuál es la justificación para alguna de sus medidas. 
El primer aspecto es de naturaleza formal y afecta a cómo se aprueban estos Tratados en el seno de la Unión Europea. A través del reconocimiento de la política comercial se ha producido una redistribución de competencias entre los Estados y la Unión Europea que ha reducido considerablemente los poderes de los Estados, sobre todo como consecuencia de la vis atractiva que está teniendo la política comercial en el ámbito comunitario. Incluso dentro de la propia estructura europea, durante la negociación del CETA y del TTIP se ha visto claramente que, desde un punto de vista político, Cecilia Malmström, responsable de la Comisaría de Comercio en la Comisión Europea es la que tiene un papel más relevante.

En efecto, la política comercial constituye el hilo conductor de estos Tratados. Se ha considerado que la regulación económica y la fijación de estándares técnicos, sociales y ambientales, dado que afecta a la distribución de bienes y servicios está incluida en su ámbito de aplicación. Una visión que, desde mi punto de vista, es demasiado expansiva y que permite eliminar toda la regulación que pueda poner en discusión el comercio internacional. Supone una aplicación de nuevos modos de regular: al igual que en Nueva Zelanda el Derecho de la competencia constituyó la regulación transversal, en la actualidad se pretende que estos tratados asuman este rol. Lo cual tiene consecuencias sobre los valores comunitarios y sobre la propia distribución de poderes entre los Estados y Bruselas.

Nótese que una visión expansiva del fomento del comercio internacional, como la que está aplicando la Unión Europea, puede entrar en colisión con principios claves del funcionamiento comunitario, como puede ser el principio de precaución. Y, desde la perspectiva nacional, poner en duda reglas derivadas de la cláusula del Estado social. En este sentido no es una cuestión, de nuevo, de proteccionismo ni de nacionalismo sino de ponderación de reglas, valores y principios.

Pero sus consecuencias no se quedan sólo aquí. A través suyo, se ha producido, por ejemplo, una reducción de los poderes de los Estados a la hora de la ratificación del CETA, debido esencialmente a su aplicación provisional y a los efectos jurídicos que tiene su consideración como Tratado mixto, en la medida en que no se exige la unanimidad de los Estados europeos. Posiblemente constituya la respuesta indirecta a la dificultad que pue- 
de suponer poner de acuerdo a los 27 Estados de la Unión. Esta reducción se ha hecho a través de dos vías.

Como paso previo, se ha producido la categorización del CETA como acuerdo mixto por razones políticas ${ }^{3}$. En el ámbito europeo supone una novedad que marca una línea entre los Tratados con Vietnam o Corea y éste. Una forma de eludir el coste político de su debate nacional en todos los Estados miembros y que resulta previsible que sea lo que se haga si ha de ser aprobado el TTIP o el TiSA. Esto tiene una consecuencia importante sobre el proceso de aprobación y otra en relación con los efectos.

Desde el primer punto de vista no es precisa la unanimidad, sino que resulta suficiente la mayoría cualificada, esto es un mínimo del $55 \%$ de los miembros del Consejo que incluya al menos a quince de ellos y represente a Estados miembros que reúnan como mínimo el $65 \%$ de la población de la Unión.

El segundo aspecto es, en mi opinión, más importante: las autoridades comunitarias plantearon que se aplique de forma provisional, desde el momento en que se apruebe por las autoridades europeas competentes. Una posición que respalda Justin Trideau, Primer Ministro canadiense. Esta aplicación provisional afectaría a las materias de competencia exclusiva de la Unión Europea y que fuentes canadienses fijan en aproximadamente un $90 \%$ del Tratado.

Ya de entrada un porcentaje de tal magnitud deja como accesoria la participación de los Parlamentos nacionales. Algo que, por su carácter poco democrático no resulta admisible especialmente teniendo en cuenta el impacto constitucional que tiene el CETA.

3 Como ha señalado la Comisaria Malmström este paso atrás de la Comisión se produce «por razones políticas» de los Estados, ya que la Comisión lo ve de otro modo: «From a strict legal standpoint, the Commission considers this agreement to fall under exclusive EU competence. However, the political situation in the Council is clear, and we understand the need for proposing it as a 'mixed' agreement, in order to allow for a speedy signature». http://europa.eu/rapid/press-release_IP-162371_en.htm. 
Pero, en segundo lugar, surge el problema de la situación de inseguridad jurídica que introduce la Comisión: ¿entraría, por ejemplo, el mecanismo «arbitral» de resolución de conflictos sobre inversiones en esta aplicación provisional? $\mathrm{O}$ dicho de otro modo ¿realmente se puede diferenciar artículo a artículo cuál entra en competencia comercial y cuál no? Si así fuera, ¿sería un Tratado aplicable? Y a partir de aquí surgen más cuestiones ¿durante cuánto tiempo duraría una aplicación provisional que ya se avanza que sería prolongada en el tiempo? ¿qué ocurriría en el caso de que alguno de los Estados rechace la ratificación del Tratado -lo que no es descartable en los casos de Bélgica y Rumanía-?

En todo caso, toda la regulación se percibe desde su vertiente comercial, lo que va a afectar tanto al contenido como a los principios generales de la regulación, ya que se introducen criterios que sirven como filtro regulatorio que sólo tienen una orientación comercial. Luego se volverá sobre ello.

Por último quería recordar aquí el efecto post mortem de algunos TNG, que se ve claramente en el CETA: En efecto, El CETA tiene una vida despu de la hipottica denuncia futura: siempre que entre en vigor de acuerdo con los procedimientos establecidos (esto es, siempre que se traspase la fase en la que entraremos ahora de la aplicación provisional), el capítulo referente a la protección jurídica de las inversiones tendrcon lo se en el artículo 30.9.

\section{EL IMPACTO DE LOS TRATADOS DE NUEVA GENERACIÓN EN LA REGULACIÓN}

Como se viene manteniendo a lo largo de estas páginas, una de las características centrales de los Tratados de Nueva Generación es su impacto en la regulación económica. Un impacto que afecta tanto al contenido regulatorio como a los elementos de procedimiento legislativo; aspectos ambos que habrán de ser tenidos en cuenta por los legisladores nacionales a la hora de aprobar sus disposiciones. 


\subsection{EL CONTENIDO COMERCIAL EXPANSIVO DE LOS TNG}

El primer aspecto afecta al cambio de orientación de la regulación. La perspectiva comercial que late en estos tratados se extiende no sólo a los apartados relativos a la regulación del comercio, sino que se incorpora como un aspecto general. Por así decirlo, ocurre algo parecido a lo que ocurre con el Derecho de la competencia en el Derecho neozelandés que acaba constituyendo el elemento general de la regulación económica.

En el caso de los TNG que tenemos más cerca, podemos encontrar dos manifestaciones:

a) En primer lugar, dando preferencia a la protección del comercio sobre otros intereses públicos que pudieran estar en presencia. Posiblemente el caso más llamativo de lo que se está señalando es la preferencia que efectúa el TTIP con respecto a la salud pública: Sólo así se pueden interpretar dos reglas: los objetivos del capítulo son, dice el artículo 1 del mismo «facilitar el comercio entre la mayor medida posible, sin perjuicio del derecho de cada parte para proteger la vida y la salud».

En lógica con lo anterior, se dispone de forma expresa que es objetivo de este capítulo «asegurar que las medidas sanitarias y fitosanitarias de las partes no creen obstáculos innecesarios al comercio». Es especialmente significativo que en los supuestos de medidas de emergencia por razones de salud, existe una reitera limitación de su aplicabilidad para evitar que a través de estas medidas que puedan suponer un perjuicio al comercio.

A ello se añade el hecho de que, del texto que conocemos del TTIP debemos extraer una consecuencia importante de cómo está configurada la prevalencia del comercio sobre los demás intereses en presencia y que afecta a un elemento de índole interno de la decisión administrativa; al cómo se pueden imponer las barreras de acceso: ha de existir una motivación especial de que no suponen una dificultad innecesaria en el comercio. Esta regla se manifiesta en un desarrollo posterior en el que tanto desde la perspectiva del procedimiento como de los propios contenidos de la reglamentación. 
b) Como elemento complementario y general a lo anterior, nos encontramos con que se incorporan principios novedosos, incluso sobre la novedosa Ley 39/2015, del Procedimiento administrativo Común que configura ciertos «principios de buena regulación» en su artículo 129. Así, el CETA impone a las Administraciones Públicas que analicen dos principios con anterioridad a la promulgación nueva normativa, los cuales - pese a la coetaneidad- no están recogidos en ella: a) Análisis de impacto comercial o b) Análisis de la compatibilidad regulatoria como manifestación de la cooperación regulatoria.

Dicho de otro modo, la valoración del interés general que han de hacer las Administraciones Públicas a partir de los TNG se concentra esencialmente en un aspecto del interés general, que es el del impacto comercial de la regulación. Una perspectiva, admisible pero que, desde luego, no casa demasiado bien con los principios de nuestra Constitución económica en el marco de un Estado social.

\subsection{LA COOPERACIÓN REGULATORIA}

Como he señalado con anterioridad, los TNG constituyen un punto de partida, no un punto de llegada. El instrumento básico para conseguir esta finalidad es la cooperación regulatoria, que modifica esencialmente las reglas democráticas de articulación de la legislación.

La OCDE ha definido la cooperación regulatoria como «cualquier tipo de acuerdo formal o informal entre pa para promover cualquier forma de cooperación en el diseño, vigilancia, implementacin o gesti a posteriori de la regulación, con el objeto de impulsar la convergencia y la consistencia de las normas jurídicas ms all

En este sentido, la cooperaci regulatoria no es una figura novedosa, sino que se puede considerar un mecanismo que se puede considerar usual tanto en tratados multilaterales (por ejemplo, el que aparece en el seno de la Organización Mundial del Comercio) o en tratados bilaterales, como los que tienen suscritos Estados Unidos y la Unión Europea. Hay, de hecho, diversos modelos que los permiten diferenciar en función de su carácter m o menos formalizado, del grado de inters que existe en la armonización legis- 
lativa, de los ámbitos (materiales o procedimentales) en los que se desarrolle o, incluso, en los participantes, si son sólo poderes $\mathrm{p}$ o participan sujetos privados que con posterioridad van a estar afectados por la reglamentación.

En los TNG, los modelos son variados y dependen esencialmente de la identidad del grado de desarrollo económico. En el RCEP, por ejemplo, las diferencias impiden que se en un primer momento se impulse la armonización normativa. Por el contrario, en el CETA y en el TTIP, el modelo de cooperación regulatoria que se plantea es el de armonización legislativa, el cual topa con diversos problemas.

Si tomamos, por ejemplo, el capítulo del TTIP relativo a la cooperación regulatoria observaremos que el objetivo de la regulación del cap de la cooperación regulatoria está muy determinado ideológicamente: «reducir la regulación onerosa, duplicativa o divergente que afecte al comercio o las inversiones». Con ello, lo que se plantea es introducir los principios de la regulación en los EE.UU., en la práctica europea y que están muy bien recogidos en el libro de Cass Sunstein, el antiguo director de la OIRA, (Office of Information and Regulatory Affairs), la oficina regulatoria de la Casa Blanca durante el primer mandato de Obama ${ }^{4}$.

Los efectos son de anticipo legislativo pero, al mismo tiempo, también de revisión de normativa. La apertura de un procedimiento de «compatibilidad normativa» es la consecuencia directa del intercambio de información regulatoria. En principio, estos mecanismos tendrán su efecto directo sobre la legislación europea, pero no podemos olvidar que caerá en cascada sobre la legislación nacional.

Desde la perspectiva del procedimiento, se aprecia que incorporan numerosos aspectos a la regulación, para conseguir el objetivo de una regulación conjunta y unitaria.

Tomando lo señalado en los documentos filtrados del TTIP afecta a la capacidad que tiene cada una de las partes de regular un ámbito de actividad. Una restricción que se produce en la medida en que se acota el pro- 
cedimiento legislativo, a través de los siguientes elementos: 1. En primer lugar, un procedimiento de coordinación, consulta y revisión normativa que identifique aquellas disposiciones que sean contradictorias con los principios que se han señalado con anterioridad. 2. En segundo lugar, se establece una obligación entre las partes de intercambiar qué reglamentaciones se van a intentar aprobar con una perspectiva anual. 3. En tercer lugar, un deber de consulta a los posibles afectados por la reglamentación para que puedan exponer cuál es su posición sobre la reglamentación. 4. En cuarto lugar, se impone un deber de transparencia, que afecta a todo el proceso de toma de decisión: objetivos que se pretenden, alternativas consideradas, análisis técnicos y científicos que han sido considerados.... Que tiene que permitir que cada operador jurídico pueda presentar alegaciones; alegaciones que tendrán que ser publicadas. 5. Un deber de comunicación entre las partes en el momento en que se ha aprobado.

Los efectos de la cooperación regulatoria están recogidos en los tratados. Tomando uno de ellos, que es similar a lo que ocurre en los demás, puede conducir a tres conclusiones distintas:

1. El examen puede determinar que se determine el reconocimiento mutuo de legislaciones; que ocurrirá en los supuestos en los que hay principios comunes de la regulación.

2. Abrir un procedimiento de armonización legislativa en los casos en los que la armonización no resulta factible. Es, además, un instrumento previo a la regulación en los supuestos en los que se quiere promover la equivalencia normativa en ámbitos internacionales en los que ambos sean parte. Es importante tener presente esto último, sobre todo para la negociación del TISA, que se está desarrollando en la actualidad en el marco de la OMC.

3. Modificación pactada de las disposiciones, con el objetivo de «simplificar actos regulatorios en línea con principios legales o administrativos compartidos».

Nótese como se introduce un sistema de solución paritaria de discrepancias cuya finalidad es reordenar las disposiciones que tengan en cartera cada una de las partes, fuera de los cauces procedimentales de aprobación de disposiciones. 


\subsection{LA ARMONIZACIÓN COMPLEJA: REGULACIONES DERIVADAS DE PRINCIPIOS COMUNES. SU IMPACTO EN EL MODELO SOCIAL EUROPEO}

Incluso en ámbitos de economía capitalista, las diferencias en la regulación son comunes. Incluso se podría señalar que, más allá de ls reglas concretas, nos encontramos con principios distintos de la regulación.

Por ejemplo, a la hora de la liberalización, el modelo que está imponiendo EEUU es el de la lista negativa (se puede privatizar todo salvo lo que esten esta lista), mientras que la postura europea es algo más suave y quiere recoger el principio de lista positiva (sólo se liberaliza aquello que está en ella) para el rgimen de trato equivalente.

$\mathrm{O}$, en un sentido parecido, la aplicación del principio de precaución tambin es muy distinta entre EEUU y la Unión Europea. el menor valor que se proporciona al principio de precaución, que está en la esencia de la política europea en este campo. No podemos olvidar que la formulación europea conlleva el que en los casos en que se presuma un riesgo, esto supondría la prohibición de un producto aunque no exista prueba científica sobre ella. De hecho, en la regulación que se conoce del TTIP no se arbitran mecanismos suficientes para evitar los «informes interesados» sobre determinados productos, tal como ha ocurrido con el glifostato de Monsanto, aprobado por la EPA a pesar de que en general se considera «probablemente cancerígeno».

Y qué decir de la aplicación del principio de transparencia, mucho más relevante en Europa que en los EEUU y que provocará un riesgo grande de captura del regulador por las empresas a las que se quiere ordenar su comportamiento. De hecho, en la regulación que se conoce del TTIP no se arbitran mecanismos suficientes para evitar los «informes interesados» sobre determinados productos.

\subsection{LOS RIESGOS DEL PROCEDIMIENTO}

Posiblemente uno de los aspectos más controvertidos es precisamente ste. Las propuestas europeas pasan por una participación activa en el pro- 
cedimiento de concertación de la reglamentación de las empresas afectadas. $\mathrm{El}$ riesgo para la reducción del impacto del principio de precaución y para incrementar la desregulación (que luego actuaría como marco de armonización legislativa).

En este sentido, corre riesgo la concepción europea del principio de transparencia, mucho más relevante en Europa que en los EEUU y que provocará un riesgo grande de captura del regulador por las empresas a las que se quiere ordenar su comportamiento. De hecho, en la regulación que se conoce del TTIP, por ejemplo, no se arbitran mecanismos suficientes para evitar los «informes interesados» sobre determinados productos y que tan usuales son en el sistema estadounidense. Los conocimientos científicos no son neutros y que, por tanto, habríamos de rodearlos de ciertas cautelas, sobre todo teniendo en cuenta que el marco de referencia de la gestión de riesgos es muy diferente entre Estados Unidos y la Unión Europea.

El contenido conocido del TTIP nos ofrece un ejemplo interesante en esta materia. A la hora de aprobar una reglamentación sanitaria, habrá de tenerse presente evidencias científicas relevantes. Parad este mandato no va acompañado de arbitrar un mecanismo de transparencia en cuanto a los estudios que justifican las decisiones tomadas, tanto en el sentido de su contenido como de su autoría. Es especialmente relevante la necesidad de transparencia en los informes sobre los que se ha adoptado la decisión, teniendo en cuenta la experiencia de la agencia EPA estadounidense sobre el glifostato de Monsanto. Es, además, un elemento determinante para saber cuál es el peso relativo que puede tener el informe en el desarrollo del procedimiento.

\subsection{EL DERECHO A LEGISLAR POR PARTE DE LOS ESTADOS}

A ello se añade el problema de la vinculación entre los arbitrajes de inversiones y el derecho a la regulación por parte de los Estados o a la limitación en cuanto a sus efectos que proviene como consecuencia de la aplicación de las cláusulas paraguas de los acuerdos de inversiones. El hecho de que el derecho a regular estsiendo recogidos en los últimos tiempos y que, en consecuencia, por ejemplo, el CETA lo contemple, no significa en modo alguno que nos debamos olvidar de la consecuencia negativa que tiene la regulación en contra de los acuerdos de inversiones. 
En efecto, no está declarado con la misma intensidad que estos cambios legislativos no harán aparecer la responsabilidad del Estado ante empresas internacionales. En efecto, cambios legislativos o de presión fiscal están siendo llevados ante los órganos arbitrales de resolución de controversias como consecuencia de que los inversores extranjeros los consideran medidas de carácter expropiatorio.

Las cláusulas «carve-out», que permiten excepcionar del rgimen arbitral a los cambios legislativos y que teóricamente est incluidas en los acuerdos, están siendo cuestionadas en los Tribunales arbitrales. Un problema que será especialmente grave cuando entre en vigor el TTIP debido a que, por su extensión, por la dificultad de negociación y la utilización de cl vagas para llegar a acuerdos, no tienen un grado de claridad suficiente y pueden entrar en contradicción con otros elementos de los Tratados.

Sea como fuere introduce un cambio sustancial en la responsabilidad por actos legislativos, que le separa sustancialmente de la regulación prevista en el Ley 40/2015, del Sector Público, que exige la inconstitucionalidad de una norma o incluso del derecho europeo. Pensemos que aquí ni siquiera es precisa la concurrencia de la contrariedad al ordenamiento jurídico. Las denominadas «expropiaciones regulatorias» amplían de forma excepcional el ámbito del funcionamiento administrativo, cuestionando las decisiones políticas que se tomen. Y cuando hablo de política, hablo de política ambiental, de obras públicas, fiscal, de protección de trabajadores; medidas que entre nosotros no dan pie a la responsabilidad del Estado pero que en el marco de estos procedimientos arbitrales sí la conceden.

\section{LOS ARBITRAJES DE INVERSIONES Y LAS ADMINISTRACIONES PÚBLICAS}

\subsection{PLANTEAMIENTO GENERAL}

El debate en la opinión pública sobre los conflictos de inversiones se ha articulado de forma muy lineal, un sí o no al arbitraje de inversiones. De hecho, los vaivenes en la opinión pública han provocado un cambio con- 
siderable en la opinión de algunos de nuestros parlamentarios: cuando el Congreso de los Diputados ratificó el Acuerdo entre la República de Corea y la Unión Europea -que incluye un mecanismo de arbitraje de inversiones- lo hizo sin un sólo voto en contra. El debate político, la oposición creciente en ciertos ámbitos de la ciudadanía europea han provocado, incluso, la propuesta de enmienda por parte de la Unión Europea para sustituir un régimen «mal visto», el del arbitraje, por otro, que tiene mejor prensa, que constituiría una especie de Tribunal que solucione los conflictos entre inversores y Estados.

Más allá del problema político que está planteando el TTIP y su mecanismo de resolución de conflictos sobre inversiones -que no es el momento de tratar-, conviene tener presente que la reglamentación de las inversiones exteriores en los Estados no deja de ser una cuestión que es de extraordinaria importancia jurídica y se sitúa en el centro de los problemas de las Administraciones Públicas frente a los tratados comerciales de nueva generación.

La globalización ha fomentado precisamente la inversión exterior y esto se ha traducido en un incremento de los conflictos sobre los (hipotéticos) derechos que tienen los operadores económicos exteriores. Un problema que, salvo el estudio de Beltrán de Felipe de hace algunos años ${ }^{5}$, no ha sido suficientemente ${ }^{6}$ tratado por los administrativistas a pesar de que afecta a aspectos esenciales del Derecho administrativo: la capacidad regulatoria de los entes públicos, los mecanismos de control judicial y el estándar de la responsabilidad administrativa.

5 M. Beltrán de Felipe, M., «¿Puede haber responsabilidad patrimonial de las Administraciones Públicas españolas al margen de la LRJAPyPAC? El arbitraje internacional de inversiones (y el estándar universal de trato justo y equitativo) como inesperado sustitutivo de la legislación interna», en la Revista General de Derecho Administrativo, $\mathrm{n}^{\circ} 16$ (diciembre 2007).

6 Tras el artículo de Beltrán, hay que citar el de J. Cantero Martínez, J.; « Arbitraje internacional de inversiones y «Derecho a regular» de los Estados: el nuevo enfoque comunitario en la negociación del TTIP», en la Revista Española de Derecho Europeo, no 58 (2016), 13 - 45. 


\subsection{FINALIDAD ESENCIAL DE LOS TRATADOS DE INVERSIONES: PROTECCIÓN DEL INVERSOR PARA GARANTÍA DE SU INVERSIÓN FRENTE A RIESGOS DE ARBITRARIEDAD Y NACIONALIDAD EN EL TRIBUNAL}

A la hora de analizar la regulación de las inversiones exteriores y, en particular, ponderar los derechos que tienen las partes del mismo conviene volver la vista un momento sobre el origen y las finalidades que se pretenden conseguir a la hora de suscribir un tratado de protección de inversiones.

Estos tratados cumplen la finalidad de proteger al inversor extranjero del riesgo de pérdida de su inversión por acciones de los Gobiernos de los países receptores de las mismas. Una perdida que se puede manifestar tanto en acciones legislativas o administrativas que no encuentran un suficiente nivel de protección jurisdiccional debido a la falta de imparcialidad de la justicia. Esto es lo que provoca que, por un lado, se establezcan restricciones al desarrollo de la acción legislativa y administrativa que pueda tener efectos sobre las inversiones -o, para ser más exacto, establecer un régimen alternativo de responsabilidad de los poderes públicos que garantice una indemnización adecuada a la cuantía del daño provocado- y que se instauren mecanismos alternativos al jurisdiccional para proporcionar un control justo de las medidas ocasionadas.

La articulación de los Tratados de protección de inversiones de este modo, con una finalidad protectora del inversor tiene su origen en que, en gran medida estos acuerdos internacionales se suscriben entre países que presentan un alto grado de desigualdad entre sus condiciones económicas y de estabilidad política. En multitud de supuestos, el país receptor de las inversiones no era totalmente seguro y esto hace que la política exterior del Estado desarrollado se manifieste en proporcionar esa seguridad jurídica al inversor nacional.

Obviamente, a cambio de esta articulación de un régimen privilegiado para la protección de la inversión, la contrapartida estriba en que el régimen sea equivalente para el proveniente del tercer país. Una previsión que no se utilizará en demasiadas ocasiones pero que está presente como vía para garantizar a los ojos de su opinión pública que hay una equivalencia en 
el reconocimiento que se hace de que ni el sistema jurídico ni el judicial es lo suficientemente estable para atraer inversiones de terceros Estados que resultan necesarias para un desarrollo social y económico.

La filosofía de los acuerdos tiene esta base. Una base derivada de la desconfianza en las condiciones jurídicas de los Estados receptores de las inversiones que no han alcanzado un suficiente grado de desarrollo. No obstante, resulta cuando menos paradójico el intento que se está produciendo en estos momentos de extender su régimen jurídico a relaciones entre países que se encuentran en una situación de estabilidad política y económica equivalente, tal como ocurre en casos como el del TTIP, antes reseñado.

Ahora bien, ¿es razonable? Tomando de nuevo las palabras de Beltrán «me parece que la justificación del arbitraje comercial internacional (la protección frente al «riesgo político») no tiene que ver con el derecho sino con la correlación de fuerzas en la política y en la economía internacional: los países exportadores de capital imponen a los países receptores de capital, que est necesitados de IED, condiciones que, lejos de ser «neutrales», favorecen los intereses del inversor estableciendo un sistema arbitral (no jurisdiccional), privado (no público), privilegiado (no neutral ni imparcial), ad hoc (no general) y con reglas jurídicas extremadamente imprecisas, flexibles y desligadas de los ordenamientos nacionales» ${ }^{7}$.

¿Y conforme con la Constitución? Cuando el artículo 106 de la Constitución determina el marco del control de la actividad administrativa lo señala de forma muy nítida tanto en el órgano como en el Derecho aplicable: «Los Tribunales controlan la potestad reglamentaria y la legalidad de la actuación administrativa». No hay control por parte de órganos del poder judicial, cuyos miembros son «independientes, inamovibles, responsables y sometidos únicamente al imperio de la ley» sino que se sustituye por árbitros privados cuya función es la protección de las inversiones, esto es, una finalidad diferente. 
No hay, en consecuencia, imperio de la ley sino protección de una parte. No hay jueces predeterminados por la ley sino un pacto sobre jueces y derecho aplicable.

\subsection{APUNTES PARA UNA VISIÓN CRÍTICA A PARTIR DE LOS PROBLEMAS QUE PLANTEAN EN EL ÁMBITO ADMINISTRATIVO LOS ARBITRAJES DE INVERSIONES}

Los arbitrajes de inversiones resultan muy problemáticos. Si quisiramos condensar todo ello en una expresión, esta sería la privatización del rgimen jurídico del tratamiento de las inversiones, lo que, desde luego, ocasiona muchas discordancias con el rgimen previsto en la Constitución:

Tomando lo señalado por Beltrán, es un procedimiento privado, resuelto por árbitros privados y no por jueces (que por tanto no tienen los deberes propios de los jueces en su labor de administrar justicia); un procedimiento privado (con restricción de partes e incluso de documentos aplicables), en el que las resoluciones no se hacen públicas y pueden no estar motivadas. Un procedimiento, en definitiva, en el que se negocia la ley aplicable y que no permite el recurso contra las resoluciones judiciales.

a) Metodológicamente, no resulta razonable. Los mecanismos especiales de resolución de conflictos, externos a los poderes judiciales de los países, surgen cuando no existe confianza en la justicia de la otra parte. Se hacen, cuando hay un alto grado de corrupción en los órganos jurisdiccionales de la otra parte o cuando se presupone que tienen una dependencia del Gobierno del Estado que hace que no haya un "proceso con todas las garantías», tal como exige el artículo 24 de la Constitución.

Pero, ¿no es suficientemente imparcial el sistema jurisdiccional europeo o el estadounidense? El mensaje que se está mandando es contrario a generar confianza en el sistema y por ello, bastante descorazonador: el rgimen judicial europeo o el estadounidense no cumple con estándares b de protección del demandante en sus relaciones con los Estados.

b) En segundo lugar, contradice la propia doctrina del Tribunal de Justicia de la Unión Europea (manifestado en el Dictamen sobre la incor- 
poración a la Convención Europea de Derechos del Hombre) sobre el monopolio del Tribunal sobre la interpretación del Derecho europeo. Dada la competencia de la Unión en materia comercial, serán decisiones europeas las analizadas, serán los Tratados europeos los analizados y ello se realizará partiendo del propio TTIP.

En este punto es llamativo un dato: la Comisión europea ten la opción de solicitar un dictamen al propio Tribunal de Justicia sobre la compatibilidad del TTIP con los Tratados europeos, tal como ha hecho en relación con el acuerdo con Singapur. Paradójicamente, no lo ha hecho con el CETA ni tampoco parece que haya intención de solicitarlo sobre el TTIP.

c) En tercer lugar, la introducción de un sistema alternativo para la resolución de diferencias sobre inversiones, al cual no pueden acceder todas las personas, lo transforma en un sistema discriminatorio; creando las denominadas «discriminaciones inversas» en las que el nacional es de peor derecho. Es un mecanismo que sirve sólo para aquellas personas jurídicas que no están domiciliadas en el ámbito territorial del demandado. Los domiciliados en ellos no pueden acceder a estos beneficios. Introduce, en consecuencia, una discriminación inversa, en donde el damnificado es el ciudadano nacional del país.

Desde el funcionamiento administrativo, hay un factor que es importante: teniendo en cuenta estas reglas ¿dónde queda el principio de inderogabilidad singular de los reglamentos?

d) Es, en cuarto lugar, contrario a las reglas de la competencia entre operadores nacionales y extranjeros, tal como estamos viendo en la actualidad en los conflictos que han surgido en relación con la supresi de los incentivos a las energías renovables. Si se sigue configurando un sistema de justicia para la protección de inversores la situación será tal como esta: el operador económico nacional tendrá que someterse a los Tribunales de justicia y se le aplicará el derecho «com» del Estado. El coste económico de la lentitud de la justicia será asumido solo por el operador nacional.

Pero, en segundo lugar, el derecho aplicable será también diferente. El extranjero podrá optar entre someterse a los Tribunales nacionales 
(sujetos a nuestro ordenamiento propio) a los Tribunales especiales y los estará sometido a la cláusula del «trato justo y equitativo» que, en la interpretación que se le está dando en las resoluciones arbitrales, puede ser beneficioso para el operador económico. Alguno de los litigios sobre inversiones que se han visto (pienso en particular en la reclamación por un reintegro de subvenciones como consecuencia del incumplimiento de su clausulado) nos hace ver que no se trata de una solución ecuánime.

¿Estamos acaso fomentando la deslocalización por razones de derecho aplicable y de Tribunales de justicia? ¿Nos damos cuenta del efecto que puede tener, además, sobre el fomento de «paraisos fiscales» $y$ otros territorios de fiscalizad poco transparente y reducida? ¿Y en el efecto que tendrá sobre las PYMES que tendrán dificultades económicas para acceder a estos mecanismos por el coste económico?

Este aspecto tiene una notable importancia en el Derecho administrativo y la forma de determinación de su aplicación. Dicho de otro modo, la base territorial que ha estado en la base del Derecho administrativo y que está incorporada al artículo 8.1 del Código civil («las leyes penales, las de policía y las de seguridad pública obligan a todos los que se hallen en territorio español») se sustituye por una base personal en la cual se pierden derechos de los afectados.

Pensemos, por ejemplo, en que el cierre de la empresa pueda provocar un despido masivo a través de un ERE. No se podrán personar en la causa. O la aplicación de otros principios y reglas de naturaleza ambiental o social ¿van a encontrar un cauce de aparición en el procedimiento arbitral?

e) En quinto lugar, nos encontramos con que es un mecanismo para la protección de los inversores extranjeros. Ni en el TTIP (por lo que se conoce) ni en otros Tratados de protección de inversiones no hay apenas menciones a la responsabilidad de los inversores en el cumplimiento de las obligaciones con el Estado.

Más aún, el sistema de arbitraje de inversiones es sólo en una dirección, esto es, el Estado no podrá demandar en un Tribunal de esta 
naturaleza a los empresarios que hayan incumplido sus obligaciones. Pero ¿qué ocurre con los Estados? ¿Tienen la posibilidad de acceder a estos mecanismos excepcionales para demandar a los inversores incumplidores de los acuerdos de inversiones? Si tomamos como punto de referencia lo que aparece regulado en el CETA, nada más lejos de la realidad.

f) Lo más problemtambin pretenden huir de la compartimentación del derecho aplicable y se sustituyen por unas reglas comunes y que reflejan un cierto grado de imperialismo jurídico anglosajón (como, por otra parte, es común en el Derecho de la globalización econ que fomenta tanto la competencia entre ordenamientos como la utilización del Derecho anglosajón como mínimo comn denominador.

Aunque en estos procedimientos hay diversas reglas la más com es la del «trato justo y equitativo». Y en cuanto al objeto, puede ser cualquier medida que menos cabe los intereses del inversor, lo que incluye cambios normativos (podríamos recordar aquí la reclamación que se planteó contra Egipto por la elevación del salario mínimo por hora de trabajo), o incluso de planeamiento urbanístico, ya que -siguiendo el patrón estadounidense- se consideran «expropiaciones regulatorias», esto es medidas que aunque no expropian directamente limitan o privan algún inters al inversor

g) El recurso a la equidad, también utilizado en los procedimientos arbitrales introduce un amplísimo grado de indeterminación, derivado de la imposibilidad jurídica de incorporar un marco que permita concretar su contenido. 